\title{
Nonuniform micromagnetic states in thin circular dots
}

\author{
Zung-Hang Wei ${ }^{\mathrm{a}, *}$, Mei-Feng Lai ${ }^{\mathrm{a}}$, Ching-Ray Chang ${ }^{\mathrm{a}}$, N.A. Usov ${ }^{\mathrm{b}}$, \\ J.C. $\mathrm{Wu}^{\mathrm{c}}$, Jun-Yang Lai ${ }^{\mathrm{a}}$ \\ a Department of Physics and Center for Nanostorage Research, National Taiwan University, Taipei 10617, Taiwan \\ ${ }^{\mathrm{b}}$ Troitsk Institute for Innovation and Fusion Research, Troitsk, Moscow Region 142190, Russia \\ ${ }^{\mathrm{c}}$ Department of Physics, National Changhua University of Education, Changhua 500, Taiwan
}

Available online 24 April 2004

\begin{abstract}
Micromagnetic states are strongly related to sizes of nano-dots. The quasiuniform state becomes unstable and transforms to a new nonuniform state as the diameter of the permalloy circular dot exceeds some critical size. The magnetization curvature increases gradually as the diameter increases. Finally, when the diameter is larger than another critical size, the new nonuniform state becomes unstable and a vortex enters the particle. Hence the new nonuniform state plays an intermediate role in the evolution from the quasiuniform state to the vortex state as the diameter increases.
\end{abstract}

(C) 2004 Elsevier B.V. All rights reserved.

PACS: 75.60.Ch; 75.75.+a

Keywords: Micromagnetic simulation; Quasiuniform state; Vortex state

\section{Introduction}

For a thin film having lateral dimensions on the order of nanometers, both the exchange and magnetostatic energies are important in determining the magnetic structures. Hence the magnetization patterns of nano-sized particles differ from those of macroscopic particles considerably. In real systems, many factors affect the magnetization structures, such as the grain size, shape, edge roughness at the boundary, anisotropy, magnetic

\footnotetext{
*Corresponding author. Tel.: + 886-2-33665183; fax: + 8862-23639984.

E-mail address: zhwei@phys.ntu.edu.tw (Z.-H. Wei).
}

history, and so on [1]. The size and shape of the patterned elements are important in determining magnetization states. Studies have recently been carried out to investigate the influence of shapes and edge roughness of the particle on the reversal process [2-5]. The edge roughness and sharp corners might produce some field leakage or nucleation centers, which will modify the equilibrium state and change the uniformity of the switching field in single domain particles. For an elliptical particle without sharp corners, the behavior of the quasiuniform state subject to external field resembles that of a single domain ellipsoidal particle [6]. By analogy with the study of a small ellipsoidal particle, which is one of the 
simplest subjects of classical micromagnetics [7], it is useful to start from smooth and circular thin films. Therefore for simplification, we concentrate the investigation on the permalloy disk without shape anisotropy and sharp corners.

In this article, we use micromagnetic simulations to study the evolution of stable states including quasiuniform state and the new nonuniform state, which is labeled as "C state" in this article, as well as vortex state existing in thin permalloy disks of different sizes. The regions for stability of quasiuniform and the $\mathrm{C}$ states are determined.

Three-dimensional micromagnetic simulations of nano-sized permalloy disk are performed by the integration of the Landau-Lifshitz-Gilbert equation [8]. Magnetostatic interactions, exchange interactions, and anisotropy field are taken into account in our simulation. The typical parameters of permalloy film are as follows: exchange constant $A=10^{-6} \mathrm{erg} / \mathrm{cm}$, and saturation magnetization $M_{\mathrm{s}}=800 \mathrm{emu} / \mathrm{cm}^{3}$. The uniaxial anisotropy constant $K_{1}$ is $10^{3} \mathrm{erg} / \mathrm{cm}^{3}$ [9], which is too small to make any influence on the total energy. The external field is absent in our study. We define the exchange length to be $R_{0}=C^{1 / 2} / M_{\mathrm{S}}=17.7 \mathrm{~nm}$. The disks are discretized into many small cubic cells, and the side length of each cubic cell is set to be lower than $3 \mathrm{~nm}$, which is much smaller than the exchange length of the permalloy, to assure the precision of our simulation. In our study, the thickness of the disks is $24 \mathrm{~nm}$ so there are eight layers of cubic cells. We set the initial magnetization to be the quasiuniform state. When the largest magnetization variation between successive iterations is below the value $10^{-8}$, the system is assumed to reach the equilibrium state.

Fig. 1 shows the typical stable states of the permalloy disks with different diameters but the same thickness. At small sizes, the quasiuniform state (Fig. 1(a)) is undoubtedly the most stable state because the exchange energy dominates. The quasiuniform state remains stable for diameters smaller than $66 \mathrm{~nm}$ and its average magnetization of the quasiuniform state is almost unity. The quasiuniform spin configuration becomes unstable and transforms into a $\mathrm{C}$ state (Fig. 1(b)) on further increase of the diameter. This $\mathrm{C}$ state has a $\mathrm{C}$ -

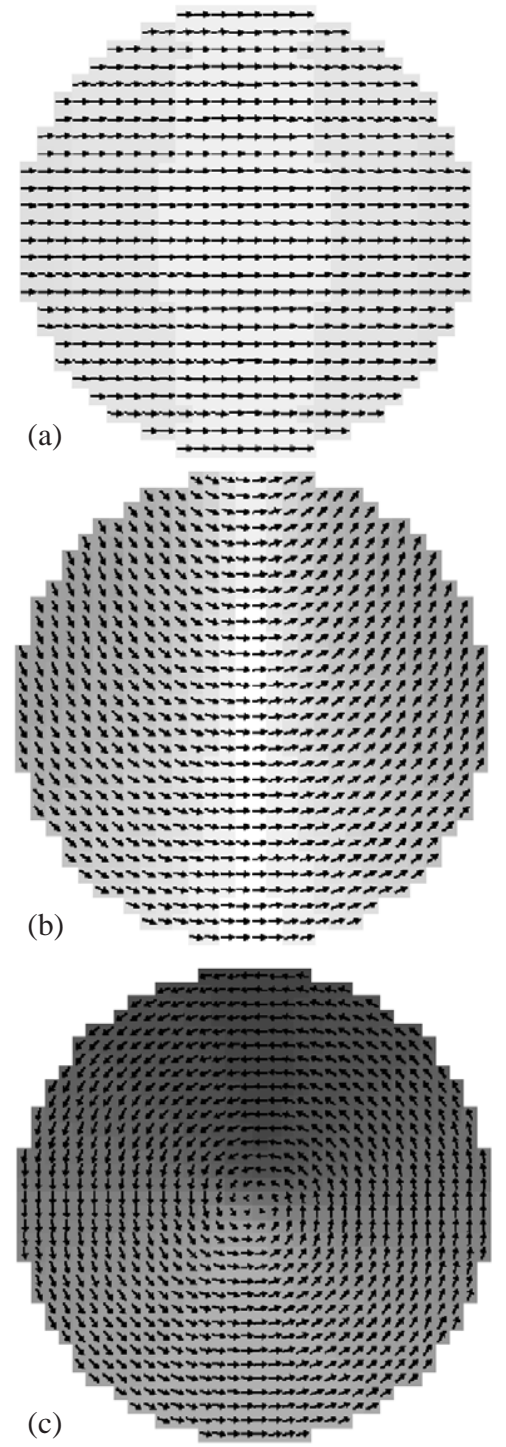

Fig. 1. Typical reduced spin configurations of permalloy disks obtained by numerical simulation. (a) is the quasiuniform state, (b) is the C state, and (c) is the vortex state. Their thickness are all $24 \mathrm{~nm}$, but the diameters are 52, 90 and $102 \mathrm{~nm}$ for (a), (b) and (c), respectively. The bright/dark color in the gray scale figures indicates the positive/negative horizontal component of the magnetization.

shaped magnetization, and the curvature of the magnetization becomes larger as the diameter increases. The average magnetization of the $\mathrm{C}$ state decreases gradually as the diameter increases because the curvature of the magnetization 
becomes more and more evident. The average magnetization decreases gradually from initial value 0.99 of the quasiuniform state to 0.77 of the $\mathrm{C}$ state. When the diameter increases to $96 \mathrm{~nm}$, the curvature of magnetization becomes too large and the instability happens. This new instability occurs via the nucleation of a vortex core near the upper edge of the particle, where the curvature of magnetization is largest. Finally, the flux closure magnetization curling replaces the original $\mathrm{C}$ shaped nonuniform spin configuration and then the disk enters a stable vortex state (Fig. 1(c)). For the vortex state the average magnetization is nearly zero.

Fig. 2 shows the energy density of different stable states as functions of disk's diameter. Obviously, the quasiuniform state has the lowest energy density for small sizes, where the vortex state has higher energy. As the diameter increases, the energy density of the vortex state decreases faster than that of the quasiuniform state. The energy densities for the quasiuniform and the vortex states are equal when the diameter reaches $46 \mathrm{~nm}$. For sizes larger than $46 \mathrm{~nm}$, the energy density of the quasiuniform state is higher than that of the vortex state. The occurrence of the $\mathrm{C}$ state causes an evident reduction of the magnetostatic energy along with a smaller increase in the exchange energy, so the total energy is reduced.

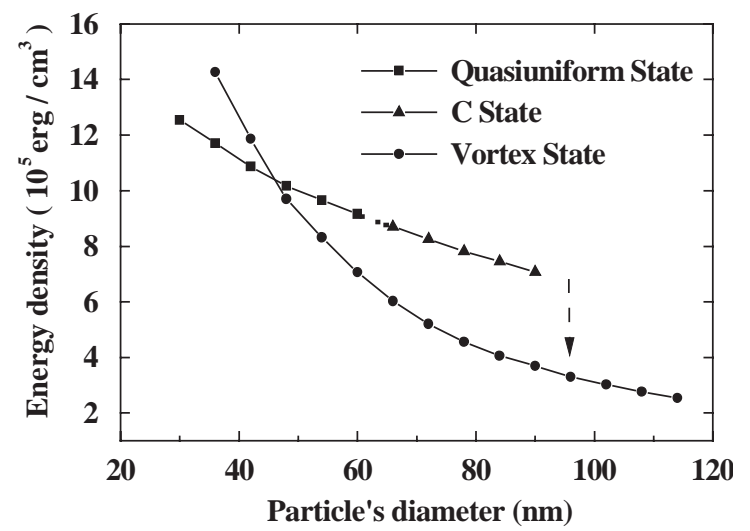

Fig. 2. Energy density versus particle's diameter for stable magnetization states in thin permalloy disks with thickness of $24 \mathrm{~nm}$. The dashed line indicates the transition region from the quasiuniform state to the $\mathrm{C}$ state. The dashed arrow indicates the transition from the $\mathrm{C}$ state to the vortex state.
Therefore, beyond some critical diameter, the $\mathrm{C}$ state becomes energetically more favorable and it is developed from a slight adjustment in spin configuration of the quasiuniform state. As the diameter continues to increase, the energy barrier between the $\mathrm{C}$ state and the vortex state is gradually reduced. The energy barrier vanishes when the diameter is larger than $96 \mathrm{~nm}$, at which the $\mathrm{C}$ state becomes unstable and transforms to the vortex state.

We illustrate the whole process by the schematic picture of the energy landscapes for possible magnetization configurations as shown in Fig. 3. When the diameter of the disk is very small, only quasiuniform state and vortex state can exist in the energy landscape. Note that, for conciseness, the quasiuniform state with reversed magnetization is not depicted here. For small disk sizes, the energy of the quasiuniform state is lower than that of the vortex state (Fig. 3(a)); however, when the disk size increases, the energy of the quasiuniform state gradually exceeds that of the vortex state (Fig. 3(b)). When the disk size reaches a critical value, the instability of the quasiuniform state occurs and

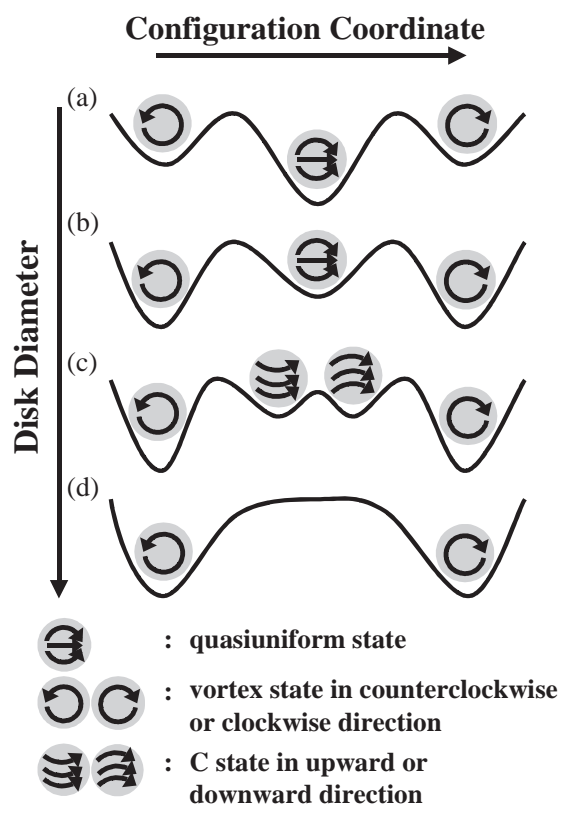

Fig. 3. The schematic picture of the energy landscapes for possible magnetization configurations as the disk diameter increases. 
the energy minimum of the quasiuniform state splits into two minimums corresponding to upward and downward $\mathrm{C}$ states (Fig. 3(c)). When the disk size increases further, the two local minimums of the $\mathrm{C}$ states disappear, and each $\mathrm{C}$ state transits to its neighboring vortex state. At last only the vortex states can exist (Fig. 3(d)).

When the temperature is taken into account, the critical diameter at which the $\mathrm{C}$ state transforms to the vortex state is reduced because the thermal fluctuations provide the energy for the $\mathrm{C}$ state to cross the small energy barrier between the $\mathrm{C}$ state and the vortex state. Further investigation of the thermal effect on the critical sizes of these stable states is needed in the future.

This work was supported in part by the R.O.C. National Science Council under Grant NSC
92-2112-M002-050 and the Technology Development Program for Academia under Grant 92-EC17-A-08-S1-0006.

\section{References}

[1] L. Thomas, et al., Appl. Phys. Lett. 76 (2000) 766.

[2] J. Shi, et al., Appl. Phys. Lett. 74 (1999) 2525.

[3] T. Schrefl, et al., J. Magn. Magn. Mater. 175 (1997) 193.

[4] H. Kronmüller, et al., J. Magn. Magn. Mater. 215 (2000) 11.

[5] M. Herrmann, et al., J. Appl. Phys. 87 (2000) 2994.

[6] N.A. Usov, et al., J. Appl. Phys. 89 (2001) 7591.

[7] W.F. Brown Jr., Micromagnetics, Interscience, New York, 1963.

[8] M.E. Schabes, J. Magn. Magn. Mater. 95 (1991) 249.

[9] I.A. Campbell, et al.,in: E. P. Wohlfarth (Ed.), Ferromagnetic Materials, Vol. 2, North-Holland, Amsterdam, 1980. 